\title{
Eye Tracking como Estratégia Avaliativa e de Ensino na Educação Inclusiva: Aplicação com Alunos com Autismo
}

\author{
Tardelly Cavalcante ${ }^{1,2}$, Jordão Frazão ${ }^{1}$, Anselmo Paiva ${ }^{3}$, Ivana Maia ${ }^{4}$ \\ Priscila Benitez $^{5}$, André Soares ${ }^{1}$ \\ ${ }^{1}$ Universidade Federal do Piauí (UFPI) - Teresina - PI - Brasil \\ ${ }^{2}$ Instituto Federal do Piauí (IFPI) - Campo Maior - PI - Brasil \\ ${ }^{3}$ Universidade Federal do Maranhão (UFMA) - São Luís - MA - Brasil \\ ${ }^{4}$ Instituto Federal do Maranhão (IFMA) - São Luís - MA - Brasil \\ ${ }^{5}$ Universidade Federal do ABC (UFABC) - Santo André - SP - Brasil \\ \{tardellycavalcante, jordaofrazao\}@gmail.com, paiva@deinf.ufma.br \\ ivana.maia@ifma.edu.br, pribenitez@yahoo.com.br, andre.soares@ufpi.edu.br
}

\begin{abstract}
In the area of education, professionals teach and evaluate the academic repertoire of students with the most diverse skills and histories. Students may experience learning difficulties, failure to adapt to pedagogical methods or other factors. To get around this, it is important to find the people who are present in the profile of each of the students. The article uses an ocular behavior analysis (eye tracking) of students during a non-computerized educational activity. For evaluation and prepared this proposal, we investigated 4 different companies that were solved by 7 students with diagnosis of Autism Spectrum Disorder - ASD.
\end{abstract}

Resumo. Na área de educação, profissionais ensinam e avaliam o repertório acadêmico de estudantes com as mais diversas habilidades, limitações $e$ históricos. Alguns desses alunos podem apresentar dificuldades de aprendizagem, diante dos métodos convencionais de ensino. Para contornar isso, é importante encontrar metodologias que se encaixem com o perfil de cada um dos estudantes. Este artigo propõe usar a análise do comportamento ocular (eye tracking) dos estudantes durante a realização de atividades educacionais no computador. Para avaliação e demonstração desta proposta, foram desenvolvidas 4 atividades diferentes que foram resolvidas por 7 estudantes com diagnóstico de Transtorno do Espectro Autista - TEA.

\section{Introdução}

O Transtorno do Espectro Autista (TEA) é um transtorno do neurodesenvolvimento caracterizado por prejuízos na capacidade de interagir e comunicar-se. Estas dificuldades comprometem o desenvolvimento de aspectos relacionados à comunicação social, bem como comportamentos estereotipados e interesses fixos e restritos. O TEA manifesta- se de forma singular em cada criança incluindo potencialidades e limitações. Segundo The Verbal Behavior Milestones Assessment and Placement Program (VBMAPP) [Sundberg 2008], existem 24 limitações de aprendizagem. A identificação dessas 
VIII Congresso Brasileiro de Informática na Educação (CBIE 2019)

Anais do XXX Simpósio Brasileiro de Informática na Educação (SBIE 2019)

limitações e a realização de terapias e atividades visando superá-las são fundamentais para a independência e autonomia de cada indivíduo. Para trabalhar nessas limitações, a estratégia mais comprovada cientificamente no ensino de indivíduos com TEA é a Análise do Comportamento Aplicada (ABA - Applied Behavior Analysis [Cooper et al. 2007].

O uso de computadores e dispositivos eletrônicos nas pesquisas voltadas ao TEA expandem-se em ritmo acelerado, conforme afirma [Shic and Goodwin 2015]. Um dispositivo que tem sido comumente utilizado se refere ao eye tracking (rastreamento ocular), que é um método não invasivo que realiza o rastreamento ocular, tais medidas são difíceis se obter utilizando métodos tradicionais [Karatekin 2007]. O rastreamento ocular codifica informações sobre atenção, controle oculomotor e fatores psicológicos dos indivíduos [Duan et al. 2018]. O uso de eye tracking para captura e análise do comportamento ocular tem crescido nos últimos anos, focado principalmente no campo da psicologia, como também é promissora no rastreio do processo cognitivo de aprendizagem [Lai et al. 2013].

Este artigo propõe o uso do eye tracking como estratégia de ensino para auxiliar para a avaliação do comportamento ocular de estudantes durante a resolução de atividades educacionais digitais. Após a captura desses dados, o profissional educador pode analisá-los, viabilizando a identificação de limitações existentes para o aprendizado desses estudantes. Com isso, o profissional educador pode adaptar as estratégias das atividades, direcionando o ambiente digital para as reais necessidades específicas de cada estudante.

\section{Trabalhos Relacionados}

O uso de computadores e dispositivos digitais têm sido apresentados como eficazes no ensino de pessoas com TEA [Silva et al. 2017], e alguns trabalhos visam esse objetivo. O uso de eye tracking também tem crescido, seja em pesquisas com TEA ou com pessoas sem TEA. Alguns trabalhos utilizam eye tracking no auxílio ao diagnóstico do TEA, como mostram as pesquisas de [Vargas-Cuentas et al. 2017] e [Fujioka et al. 2016].

Em [Junior et al. 2017] foi utilizado o rastreio ocular na avaliação da aprendizagem, a partir de jogos digitais. A avaliação foi realizada com os dados gerados pelo eye tracking de estudantes durante a execução de jogos digitais. Em [Almourad et al. 2018], os autores utilizaram os dados do eye tracking para analisar e comparar o comportamento de indivíduos com e sem TEA enquanto olhavam para diferentes estímulos, dentre eles o rosto humano. Os autores em [Duan et al. 2018] coletaram dados via eye tracking de crianças com TEA para criar uma base de dados, contendo os pontos em que indivíduos com TEA olharam em cada situação apresentada.

Em [Moore et al. 2018] usaram eye tracking para verificar que um subgrupo de crianças com TEA, quando expostas a imagens sociais e geométricas, tiveram preferências nas imagens geométricas. Já em [Giordano et al. 2017] foi proposto um sistema computacional integrados com o eye tracking que permite aos profissionais aplicarem testes , como o teste de déficit de atenção utilizando rastreamento ocular.

As medidas de eye tracking, em geral, foram utilizadas em estudos anteriores para fins de avaliação em [Mercadante et al. 2006] e [Schwartzman et al. 2015], sobretudo neuropsicológica [Orsati et al. 2008]. Através de tais medidas é possível avaliar se o estudante apresentou, por exemplo, fixações oculares para os estímulos corretos antes de selecioná-los, se está sob controle dos estímulos distratores, fornecendo indicativos mais 
VIII Congresso Brasileiro de Informática na Educação (CBIE 2019)

Anais do XXX Simpósio Brasileiro de Informática na Educação (SBIE 2019)

precisos sobre o controle de estímulos do comportamento de observação do estudante e assim, replanejar as tarefas de ensino de maneira mais personalizada, visando a aprendizagem individual de cada um deles. Por este motivo, acredita-se que ao medir, por exemplo, o rastreamento ocular durante a intervenção educacional, como medida adicional, pode ser um passo importante na garantia de procedimentos de ensino mais personalizados para cada estudante.

Dessa forma, este artigo propõe o uso do eye tracking para auxiliar o profissional a entender o comportamento ocular do estudante durante a realização de tarefas no computador. Com isso é possível medir com precisão os aspectos relacionados ao comportamento do olhar, potencializando a identificação das limitações que dificultam o aprendizado do estudante. A quantificação das medidas obtidas a partir do rastreamento ocular são valiosas para entender como o estudante resolve uma atividade de ensino no computador.

\section{Uso de Eye Tracking como Ferramenta na Educação Inclusiva}

Segundo a Lei Brasileira de Inclusão da Pessoa com Deficiência (Estatuto da Pessoa com Deficiência) [BRASIL 2015], educação constitui direito da pessoa com deficiência, assegurados sistema educacional inclusivo em todos os níveis e aprendizado ao longo de toda a vida, de forma a alcançar o máximo desenvolvimento possível de seus talentos e habilidades físicas, sensoriais, intelectuais e sociais, segundo suas características, interesses e necessidades de aprendizagem.

Há, entretanto, necessidades que interferem de maneira significativa no processo de aprendizagem e que exigem ações educativas específicas dos profissionais da área como, por exemplo, a utilização de recursos e apoios especializados para garantir a aprendizagem de todos os alunos. Algumas dessas dificuldades podem ser mais facilmente identificadas analisando o comportamento do eye tracking para definição de novas estratégias de ensino.

Este trabalho propõe o uso do eye tracking para fornecer medidas precisas do comportamento ocular dos estudantes durante a realização de tarefas no computador. Para demonstrar esta proposta, este artigo apresenta a análise de atividades digitais usando o eye tracking. Esses dados são apresentados em gráficos que potencializam as intervenções dos profissionais junto ao estudantes conforme suas necessidades específicas. Neste contexto, foram desenvolvidas 4 atividades digitais para serem resolvidas pelos estudantes com aquisição das informações através do eye tracking. Os dados do rastreamento ocular podem ajudar a explorar a capacidade dos estudantes de categorizar eventos visuais, linguístico e examinar objetos dinâmicos.

A Figura 1a ilustra a atividade de emparelhamento com o modelo (A1). Esse tipo de atividade é utilizada no ensino e avaliação de relações condicionais, ou seja relações entre estímulos, que podem dar origem à formação de classes de equivalência [Kelly et al. 1998]. Esta atividade é realizada através do emparelhamento ou associação de 4 imagens de animais com as suas respectivas sombras. A Figura $1 \mathrm{~b}$ apresenta a atividade de formação de palavra (A2), realizada através da identificação do objeto apresentado na tela, e da formação da palavra referente ao mesmo. Nessas duas atividades, tanto as letras como os animais são embaralhados com o intuito de remover viés de posição.

A Figura 2a ilustra a atividade de formação de frase (A3). Nessa atividade foi 


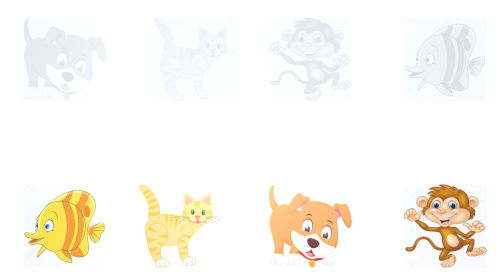

(a) Emparelhamento com o modelo

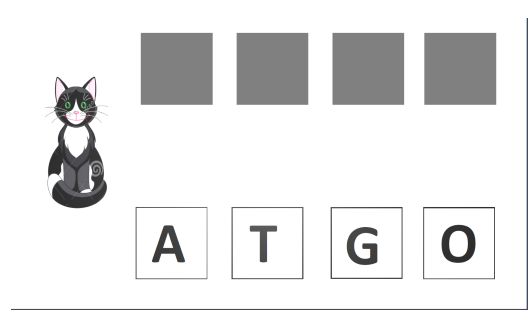

(b) Formação de Palavras

Figura 1. Exemplos das Atividades Emparelhamento com o modelo (a) e Formação de Palavras (b).

usado um objeto como exemplo da ação executada, e as palavras embaralhadas que formam a frase referentes a essa ação. A Figura $2 b$ exemplifica a atividade de identificação de frase (A4). Nessa última atividade é apresentada uma frase completa e quatro estímulos abaixo dela. $\mathrm{O}$ aluno deve ler a frase e identificar o estimulo que corresponde à frase.

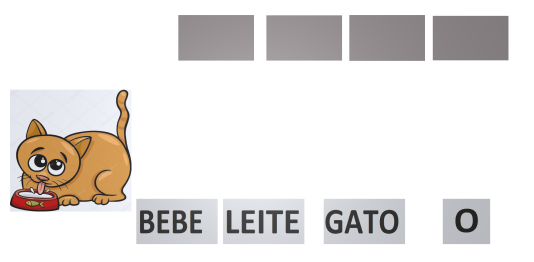

(a) Formação de Frase

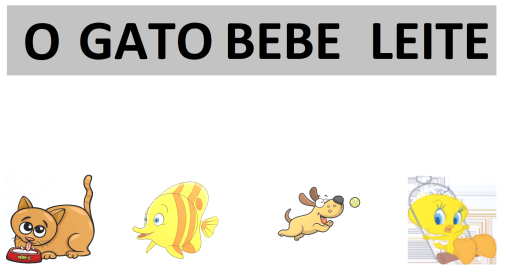

(b) Identificação de Frase

Figura 2. Exemplos das Atividades Formação de Frase (a) e Identificação de Frase (b).

Vale ressaltar que ao movimentar um componente das atividades para o destino errado o objeto volta para o local original. Assim, o estudante somente consegue movimentar definitivamente cada componente das atividades se for para o local de destino correto.

\section{Metodologia}

Foram selecionados 7 (sete) estudantes de uma escola municipal de ensino, 5 (cinco) do sexo masculino e 2 (duas) do sexo feminino. Todos os participantes com alguma especificidade diagnosticada clinicamente por profissional experiente baseando- se nos critérios do Diagnostic and Statistical Manual of Mental Disorders [Association et al. 2013]. Os 7 estudantes conseguiram realizar o procedimento de calibracão, exigidos pelo eye tracking. Todos os participantes tiveram os termos de consentimento assinados pelos respectivos responsáveis.

Os experimentos foram realizados durante o Atendimento Educacional Especializado - AEE, no contraturno ao período escolar em que os estudante estavam matriculados. O professor do AEE acompanhou todo o processo de aplicação das atividades, em conjunto ao pesquisador. A aplicação foi individualizada com cada estudante com TEA, de modo a garantir a situação naturalística de ensino prevista no AEE. No caso do presente estudo, o AEE é oferecido individualmente para cada estudante, no contraturno escolar. 
Inicialmente foi realizado um processo de calibração, no qual os participantes ficaram sentados em frente ao computador a uma distância de $50 \mathrm{~cm}$, olhando frontalmente para a tela. Em seguida, atividades em forma de jogos digitais foram apresentadas sequencialmente aos estudantes. Durante a execução das atividades foram rastreados os dados do olhar de cada participante em forma de coordenadas $(x, y)$ através de uma câmera e processamento de imagem.

Após esse processo de execução das atividades foram processadas as informações do rastreio ocular de cada participante individualmente com uso das bibliotecas Pygaze [Dalmaijer et al. 2014] e Matplotlib do Python. Esses dados foram utilizados para identificação de informações como fixações, sacadas e interações relacionais entre o eye tracking e o mouse.

\section{Resultados}

A Figura 3 apresenta o mapa de calor referente a dois comportamentos oculares diferentes. O mapa de calor são relatórios indicando quais as áreas da tela foram mais observadas pelo estudante. $\mathrm{O}$ aumento da intensidade de calor indica maior observação.

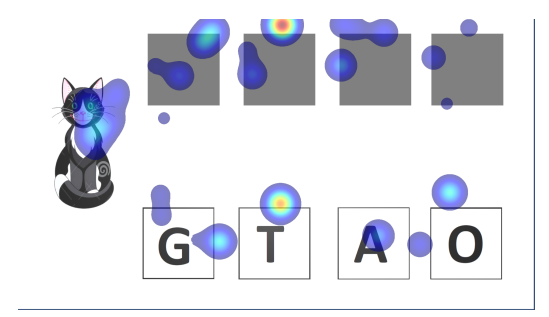

(a) Execução com foco no Estimulo

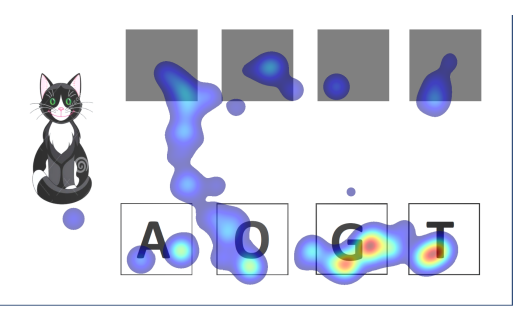

(b) Execução sem foco no Estímulo

Figura 3. Mapa de calor do Estudante 5 (a) e do Estudante 2 (b)

Na Figura 3a, analisando o mapa de calor, percebe-se que o estudante observou o estímulo apresentado pela tarefa, a imagem do gato. Percebe-se pela Figura 3a que o estímulos planejados pelo profissional educador chamou a atenção do Estudante 5. Por outro lado, a Figura 3 b mostra por meio do mapa de calor que o Estudante 2 não olhou atentamente para a imagem do gato. Dessa forma, o profissional educador fica ciente que o Estudante 2 não apresentou interesse na imagem do gato inserida na tarefa. Vale ressaltar que o Estudante 2 pareceu demonstrar pouco interesse pela atividade, o que significou que talvez o ambiente não tivesse contingências reforçadoras suficientes para garantir o interesse na atividade.

A Figura 4 apresenta o movimento do olhar e do mouse durante a resolução da atividade A2, formação da palavra gato, realizada pelo estudante 5. Na parte superior da Figura 4 observa-se a movimentação do olhar (linha azul) e do mouse (linha laranja) para cada uma das 4 letras. Na parte inferior da Figura 4 (linha preta) apresenta-se o tempo de movimentação de cada uma das letras, no processo de formação da palavra gato. Cada gráfico da parte inferior refere-se à movimentação de uma das letras. No eixo X está o tempo decorrido durante toda a atividade, e no eixo $\mathrm{Y}$, com valores 1 (um) para o tempo utilizado na movimentação de cada uma das letras. $\mathrm{O}$ valor 0 (zero) indica que não houve movimentação da respectiva letra. 

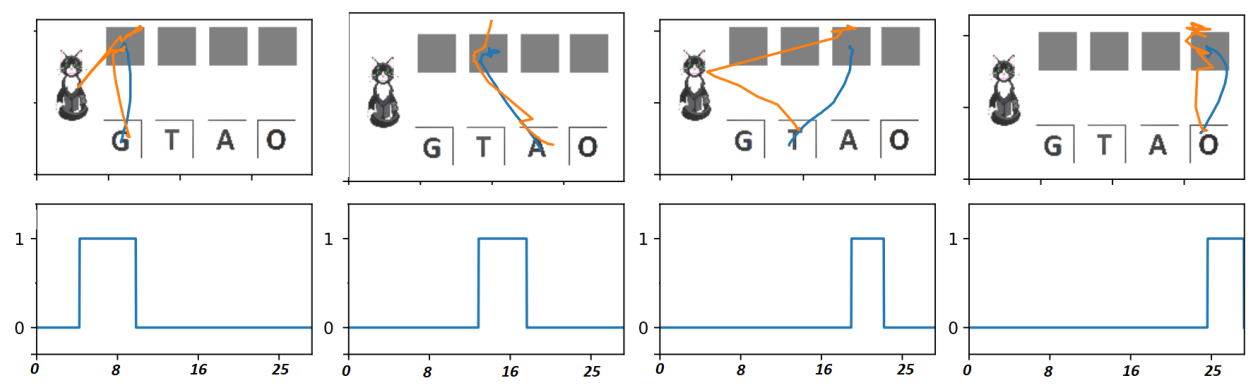

Figura 4. Execução segmentada da atividade formar palavra gato com foco no estimulo, estudante 5 .

Ao analisar a Figura 4 o profissional educador sabe que o Estudante 5 movimentou primeiro a letra $\mathrm{G}$ e gastou cerca de 5 segundos para mover a letra "G" para o local correto. Em seguida o Estudante 5 moveu a letra "A" gastando 4 segundos. Depois o Estudante 5 moveu as letras " $T$ " e "O" gastando respectivamente 3 e 4 segundos. Tal comportamento é esperado para os estudante que possuem a habilidade de leitura.

A Figura 5 apresenta o mesmo tipo de gráfico da Figura 4, mas referente aos resultados do Estudante 2, que não olhou atentamente na imagem do gato.
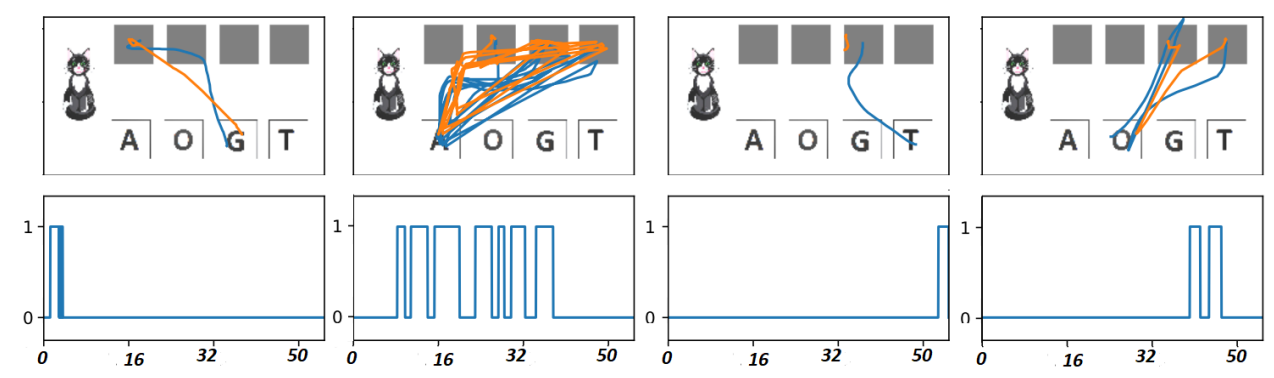

Figura 5. Execução segmentada da atividade formar palavra gato sem foco no
estímulo, estudante 2 .

Na Figura 5, ao analisar o instante de tempo no qual cada letra foi movimentada, observa-se que o Estudante 2 movimentou inicialmente de forma correta a letra " $G$ " para a primeira posição da palavra. Entretanto, observa-se também que a movimentação da letra "A" foi realizada várias vezes para todos os outros 3 espaços restantes. Isso sinaliza que o Estudante 2 movimentou a letra "A" usando um método de tentativa e erro.

Vale ressaltar que foi observado uma correlação entre o movimento do mouse do computador e do movimento do rastreio ocular. Essa correlação indica que o estudante movia o mouse e olhava a movimentação, comportamento esperado na resolução atenta de uma dada atividade no computador.

Outro dado importante são as fixações que representam dados focados em um determinado espaço de tempo em uma área ou região. A Figura 6a apresenta as fixações e sacadas na resolução da atividade de emparelhamento com o modelo, realizada pelo Estudante 5. A Figura 6 b mostra as fixações e sacadas na resolução da atividade de formação de frase, feita pelo Estudante 3. Esse gráfico é formado por circunferências verdes que indicam a área dentro de determinado raio limite onde o estudante fixou o 
VIII Congresso Brasileiro de Informática na Educação (CBIE 2019)

Anais do XXX Simpósio Brasileiro de Informática na Educação (SBIE 2019)

olhar durante um determinado período de tempo. Além das fixações, observa-se também as sacadas, representadas pelas ligações entre as sacadas, que são movimentos rápidos entre fixações [Salvucci and Goldberg 2000]. Ainda é possível analisar o trajeto entre as fixações indicando o caminho percorrido pelo olhar do estudante durante a realização da atividade.

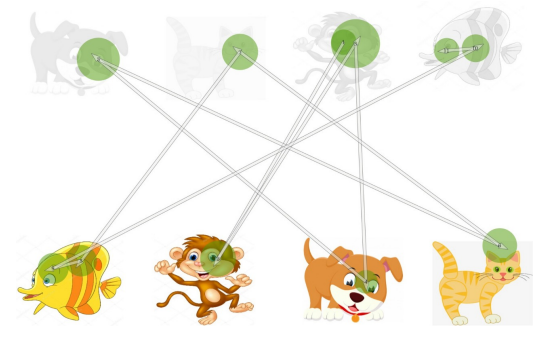

(a) Atividade 1 .

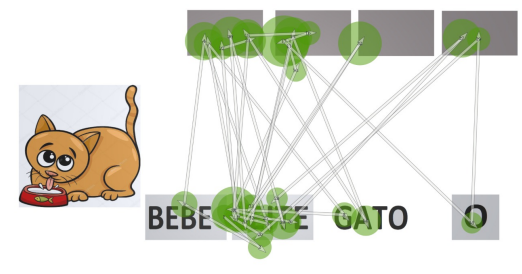

(b) Atividade 3 .

Figura 6. Fixações e sacadas.

A Figura 6a apresenta fixações bem alinhadas com os estímulos propostos na tarefa. Além disso, nota-se que os movimentos sacádicos foram correspondentes à movimentação entre as imagens e os seus respectivos destinos, indicando que a atividade foi bem entendida pelo estudante, uma vez que os movimentos dos olhos corresponderam ao que se espera durante a realização da atividade proposta.

A Figura $6 \mathrm{~b}$ mostra um número maior de fixações e sacadas, se comparada com a Figura 6a. Isso já demonstra que a atividade de formação de frase é mais complexa do que a de emparelhamento de formas, conforme esperado. Essa informação pode demonstrar indícios de como e quais atividades serão as mais adequadas ao estudante. Esse tipo de análise pode ser útil para identificar que a atividades é complexa para o repertório do aluno, apresentando uma grande quantidade de fixações na área onde não foi possível identificar o que deve ser feito na atividade.

De maneira geral, percebeu-se maior dificuldade na execução na tarefa de formação de frase, onde os estudantes levaram mais tempo para resolvê-las. A Figura 7 mostra o comportamento do mouse do Estudante 6 que demonstrou boa capacidade na resolução da atividade de formação de frase.

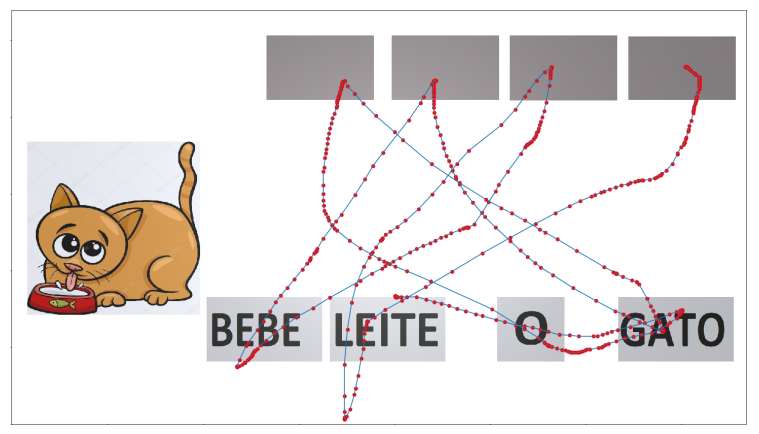

Figura 7. Movimentação do mouse.

Os estudantes que demonstraram maior habilidade em resolver a atividade A3, apresentam na movimentação do mouse geralmente duas arestas em cada componente da 
VIII Congresso Brasileiro de Informática na Educação (CBIE 2019)

Anais do XXX Simpósio Brasileiro de Informática na Educação (SBIE 2019)

atividade, como demonstrado na Figura 7. Tais informações sugerem que o estudante já possui repertório para resolver essa atividade e assim, pode-se definir novas tarefas de maior desafio.

Dados como a porcentagem do tempo em que o estudante mantém o olhar na tela também podem ser interessantes aos profissionais, pois é possível identificar a presença de distratores no ambiente ou outras limitações durante a resolução da atividade proposta.

A Figura 8 relaciona o tempo necessário para resolução de cada atividade para cada estudante.

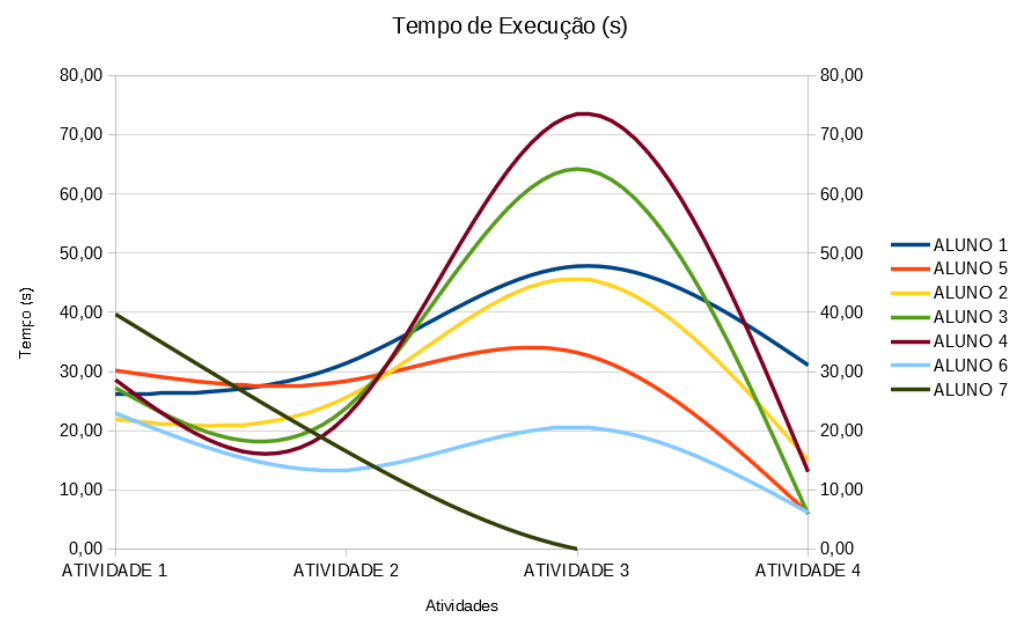

Figura 8. Relação do tempo de execução entre atividades de cada estudante.

De maneira geral, nota-se na Figura 8 que a relação do tempo gasto para a resolução de cada uma das atividades apresentou comportamento similar entre os estudantes. Isso foi observado apesar das diferentes especificidades de cada um dos estudantes e de seus diferentes repertórios. Percebe-se que a atividade A3 (formação de frase) foi aquela que demandou mais tempo dos estudantes, devido a sua maior complexidade.

O Estudante 7 não resolveu as atividades A3 e A4, respectivamente de formação de frase e identificação de frase. A situação experimental não foi reforçadora o suficiente para garantir a realização do experimento com estudante 7 .

Detecta-se ainda que no geral, houve um decréscimo do tempo de resolução significativo entre a atividade A3 e A4. Acredita-se que essa redução é justificada pela similaridade dos dados entre as atividades A3 e A4. Essas duas atividades foram construídas com base na ação "o gato bebe leite". Tal comportamento sugere a aquisição do conhecimento no decorrer das resoluções de atividades anteriores.

\section{Conclusões}

Este trabalho apresentou a proposta de uso do eye tracking como ferramenta auxiliar na avaliação e planejamento de atividades de ensino no computador. Com isso o profissional educador passa a ter medidas precisas do comportamento ocular dos estudantes durante a realização de tarefas no computador. Conforme discutido ao longo do trabalho, o comportamento ocular dos estudantes pode ser observados em gráficos que potencializam as intervenções dos profissionais junto aos estudantes conforme suas necessidades 
VIII Congresso Brasileiro de Informática na Educação (CBIE 2019)

Anais do XXX Simpósio Brasileiro de Informática na Educação (SBIE 2019)

específicas. A análise destes dados potencializa a identificação de limitações de aprendizagem dos estudantes nessas atividades, bem como possíveis atividades já generalizadas por eles. Além disso, a aplicação da pesquisa mostrou o uso do eye tracking, em situação naturalística de ensino, por meio da aplicação durante o AEE, em situação escolar.

Essa proposta está em consonância com a Análise do Comportamento Aplicada, que sugere a personalização das tarefas do indivíduo com base no seu desempenho e currículo, incrementando cada vez mais o seu aprendizado. Outro fator importante se refere ao feedback dado pelo profissional educador, após apresentação dos resultados das aplicações, no sentido de que tais análises puderam confirmar que os estudantes estavam olhando para o computador para realização das atividades, além de estarem sob controle das instruções e dos estímulos apresentados para a realização das tarefas, o que significa que eles estavam prestando atenção nas instruções dadas para emissão de cada resposta.

Como trabalhos futuros, pretende-se evoluir a proposta para incluir reforçadores imediatos e personalizados entre cada atividade, a fim de aumentar ainda mais o interesse dos estudantes. Além disso, pretende-se ampliar o experimento a outros públicos, como também em crianças sem nenhum tipo de transtorno ou deficiência, para verificar o desenvolvimento das mesmas, focando o uso do eye tracking na perspectivava do desenho universal. Foram realizados testes iniciais com crianças com síndrome de Down e Esquizofrenia e verificou-se que a proposta é inclusiva e pode alcançar um grande público de estudantes.

\section{Referências}

Almourad, M. B., Bataineh, E., Stocker, J., and Marir, F. (2018). Analyzing the behavior of autistic and normal developing children using eye tracking data. In International Conference on Kansei Engineering \& Emotion Research, pages 340-349. Springer.

Association, A. P. et al. (2013). Diagnostic and statistical manual of mental disorders (DSM-5@). American Psychiatric Pub.

BRASIL (2015). Lei brasileira de inclusão da pessoa com deficiência.

Cooper, J. O., Heron, T. E., Heward, W. L., et al. (2007). Applied behavior analysis.

Dalmaijer, E. S., Mathôt, S., and Van der Stigchel, S. (2014). Pygaze: An open-source, cross-platform toolbox for minimal-effort programming of eyetracking experiments. Behavior research methods, 46(4):913-921.

Duan, H., Zhai, G., Min, X., Fang, Y., Che, Z., Yang, X., Zhi, C., Yang, H., and Liu, N. (2018). Learning to predict where the children with asd look. In 2018 25th IEEE International Conference on Image Processing (ICIP), pages 704-708. IEEE.

Fujioka, T., Inohara, K., Okamoto, Y., Masuya, Y., Ishitobi, M., Saito, D. N., Jung, M., Arai, S., Matsumura, Y., Fujisawa, T. X., et al. (2016). Gazefinder as a clinical supplementary tool for discriminating between autism spectrum disorder and typical development in male adolescents and adults. Molecular autism, 7(1):19.

Giordano, D., Pino, C., Kavasidis, I., Spampinato, C., Di Pietro, M., Rizzo, R., Scuderi, A., and Barone, R. (2017). An eye tracker based computer system to support oculomotor and attention deficit investigations. In 2017 IEEE 30th International Symposium on Computer-Based Medical Systems (CBMS), pages 538-543. IEEE. 
VIII Congresso Brasileiro de Informática na Educação (CBIE 2019)

Anais do XXX Simpósio Brasileiro de Informática na Educação (SBIE 2019)

Junior, H., de Menezes, C., and De Souza, A. (2017). Monitoramento dos movimentos dos olhos para apoiar a avaliação da aprendizagem em jogos digitais. In Brazilian Symposium on Computers in Education (Simpósio Brasileiro de Informática na Educação-SBIE), volume 28, page 787.

Karatekin, C. (2007). Eye tracking studies of normative and atypical development. Developmental review, 27(3):283-348.

Kelly, S., Green, G., and Sidman, M. (1998). Visual identity matching and auditory-visual matching: A procedural note. Journal of Applied Behavior Analysis, 31(2):237-243.

Lai, M.-L., Tsai, M.-J., Yang, F.-Y., Hsu, C.-Y., Liu, T.-C., Lee, S. W.-Y., Lee, M.-H., Chiou, G.-L., Liang, J.-C., and Tsai, C.-C. (2013). A review of using eye-tracking technology in exploring learning from 2000 to 2012. Educational research review, 10:90-115.

Mercadante, M. T., Macedo, E. C., Baptista, P. M., Paula, C. S., and Schwartzman, J. S. (2006). Saccadic movements using eye-tracking technology in individuals with autism spectrum disorders: pilot study. Arquivos de neuro-psiquiatria, 64(3A):559-562.

Moore, A., Wozniak, M., Yousef, A., Barnes, C. C., Cha, D., Courchesne, E., and Pierce, K. (2018). The geometric preference subtype in asd: identifying a consistent, earlyemerging phenomenon through eye tracking. Molecular autism, 9(1):19.

Orsati, F. T., Schwartzman, J. S., Brunoni, D., Mecca, T., and de Macedo, E. C. (2008). Novas possibilidades na avaliação neuropsicológica dos transtornos invasivos do desenvolvimento: Análise dos movimentos oculares. Avaliaçao Psicologica: Interamerican Journal of Psychological Assessment, 7(3):281-290.

Salvucci, D. D. and Goldberg, J. H. (2000). Identifying fixations and saccades in eyetracking protocols. In Proceedings of the 2000 symposium on Eye tracking research \& applications, pages 71-78. ACM.

Schwartzman, J. S., Velloso, R. d. L., D’Antino, M. E. F., and Santos, S. (2015). The eyetracking of social stimuli in patients with rett syndrome and autism spectrum disorders: a pilot study. Arquivos de neuro-psiquiatria, 73(5):402-407.

Shic, F. and Goodwin, M. (2015). Introduction to technologies in the daily lives of individuals with autism.

Silva, M., Moura, I., and Soares, A. (2017). Uso de tecnologias computacionais para o ensino de crianças com transtorno do espectro autista: Um mapeamento sistemático da literatura. In Brazilian Symposium on Computers in Education (Simpósio Brasileiro de Informática na Educação-SBIE), volume 28, page 173.

Sundberg, M. L. (2008). VB-MAPP Verbal Behavior Milestones Assessment and Placement Program: a language and social skills assessment program for children with autism or other developmental disabilities: guide. Mark Sundberg.

Vargas-Cuentas, N. I., Roman-Gonzalez, A., Gilman, R. H., Barrientos, F., Ting, J., Hidalgo, D., Jensen, K., and Zimic, M. (2017). Developing an eye-tracking algorithm as a potential tool for early diagnosis of autism spectrum disorder in children. PloS one, 12(11):e0188826. 